\title{
Article \\ A New Class of Coupled Systems of Nonlinear Hyperbolic Partial Fractional Differential Equations in Generalized Banach Spaces Involving the $\psi$-Caputo Fractional Derivative
}

\author{
Zidane Baitiche ${ }^{1}$, Choukri Derbazi ${ }^{1}{ }^{\complement}$, Mouffak Benchohra ${ }^{2}$ and Yong Zhou ${ }^{3, *}$ \\ 1 Laboratoire Equations Différentielles, Department of Mathematics, Faculty of Exact Sciences, Frères Mentouri \\ University Constantine 1, Ain El Bey Way, P.O. Box 325, Constantine 25017, Algeria; \\ zidane.baitiche@umc.edu.dz (Z.B.); choukri.derbazi@umc.edu.dz (C.D.) \\ 2 Laboratory of Mathematics, Djillali Liabes University of Sidi-Bel-Abbes, P.O. Box 89, Sidi Bel Abbes 22000, \\ Algeria; benchohra@univ-sba.dz \\ 3 Faculty of Information Technology, Macau University of Science and Technology, Macau 999078, China \\ * Correspondence: yozhou@must.edu.mo
}

Citation: Baitiche, Z.; Derbazi, C.; Benchohra, M.; Zhou, Y. A New Class of Coupled Systems of Nonlinear Hyperbolic Partial Fractional Differential Equations in Generalized Banach Spaces Involving the $\psi$-Caputo Fractional Derivative. Symmetry 2021, 13, 2412. https:// doi.org/10.3390/sym13122412

Academic Editors: Garik Petrosyan, Zine El Abiddine Fellah and Alexander Zaslavski

Received: 15 November 2021 Accepted: 7 December 2021 Published: 13 December 2021

Publisher's Note: MDPI stays neutral with regard to jurisdictional claims in published maps and institutional affiliations.

Copyright: (c) 2021 by the authors. Licensee MDPI, Basel, Switzerland. This article is an open access article distributed under the terms and conditions of the Creative Commons Attribution (CC BY) license (https:/ / creativecommons.org/licenses/by/ $4.0 /)$.

\begin{abstract}
The current study is devoted to investigating the existence and uniqueness of solutions for a new class of symmetrically coupled system of nonlinear hyperbolic partial-fractional differential equations in generalized Banach spaces in the sense of $\psi$-Caputo partial fractional derivative. Our approach is based on the Krasnoselskii-type fixed point theorem in generalized Banach spaces and Perov's fixed point theorem together with the Bielecki norm, while Urs's approach was used to prove the Ulam-Hyers stability of solutions of our system. Finally, some examples are provided in order to illustrate our theoretical results.
\end{abstract}

Keywords: $\psi$-Caputo fractional partial derivative; existence; uniqueness; fixed point; Bielecki norm; Ulam stability; generalized Banach spaces

\section{Introduction}

During the last twenty years, fractional differential equations have gained considerable attention in various fields of applied mathematics and engineering. For details and applications, we refer the reader to basic books [1-9]. Currently, there are several definitions of fractional derivatives in the literature, but the most popular ones are RiemannLiouville, Caputo, and Caputo-Hadamard. A generalization of derivatives of both Caputo and Caputo-Hadamard was provided by Almeida in [10]. He named it as a $\psi$-Caputo fractional derivative. Moreover, Sousa and Oliveira presented an interesting fractional partial derivative, which they called the $\psi$-Hilfer fractional partial derivative. An excellent account of information on this new fractional operator can be found in papers [11,12].

On the other hand, there have been a huge amount of papers that deal with the existence, uniqueness, and stability of solutions for ordinary and partial differential equations involving fractional derivatives with the help of different techniques of nonlinear analysis (see, for instance, [13-19] and the references cited therein). In addition, the study of coupled systems of fractional differential equations has also attracted considerable attention because such systems appear in various scientific applications (we refer to [20-22]). For some recent results on the existence and stability of solutions for a coupled system of fractional differential equations involving different forms of fractional derivatives, we refer the reader to [23-28]. To the best of our knowledge in the field, no one considered a coupled system of nonlinear hyperbolic partial fractional differential equations in generalized Banach spaces involving the $\psi$-Caputo fractional partial derivative. Motivated by the above-mentioned 
reason, in this paper, we study the existence, uniqueness, and the Ulam-Hyers stability of solutions for the following coupled system with symmetry:

$$
\left\{\begin{array}{l}
\left({ }^{c} \mathbb{D}_{\tilde{a}}^{\alpha ; \psi} x\right)(\tau, \mathrm{u})=\lambda_{1} x(\tau, \mathrm{u})+\mathbb{F}_{1}(\tau, \mathrm{u}, x(\tau, \mathrm{u}), y(\tau, \mathrm{u})), \\
\left({ }^{c} \mathbb{D}_{\tilde{a}}^{\beta ; \psi} y\right)(\tau, \mathrm{u})=\lambda_{2} y(\tau, \mathrm{u})+\mathbb{F}_{2}(\tau, \mathrm{u}, x(\tau, \mathrm{u}), y(\tau, \mathrm{u})),
\end{array} \quad(\tau, \mathrm{u}) \in \tilde{\mathrm{I}},\right.
$$

under the following initial conditions:

$$
\left\{\begin{array}{l}
x(\tau, a)=\eta_{1}(\tau), \quad \tau \in[a, b] \\
x(a, \mathrm{u})=v_{1}(\mathrm{u}), \quad \mathrm{u} \in[a, c] \\
x(a, a)=\eta_{1}(a)=v_{1}(a), \\
y(\tau, a)=\eta_{2}(\tau), \quad \tau \in[a, b] \\
y(a, \mathrm{u})=v_{2}(\mathrm{u}), \quad \mathrm{u} \in[a, c] \\
y(a, a)=\eta_{2}(a)=v_{2}(a),
\end{array}\right.
$$

where I $:=[a, b] \times[a, c], a, b$ and $c$ are positive constants, and $\mathbb{F}_{1}, \mathbb{F}_{2}: \tilde{\mathrm{I}} \times \mathbb{R}^{2} \longrightarrow \mathbb{R}$ are given continuous functions. $\eta_{1}, \eta_{2}:[a, b] \longrightarrow \mathbb{R}, v_{1}, v_{2}:[a, c] \longrightarrow \mathbb{R}$ are given absolutely continuous functions. ${ }^{c} \mathbb{D}_{a^{+}}^{\alpha ; \psi},{ }^{c} \mathbb{D}_{a^{+}}^{\beta ; \psi}$ are the $\psi$-Caputo fractional derivatives of order $\alpha=\left(\alpha_{1}, \alpha_{2}\right), \beta=\left(\beta_{1}, \beta_{2}\right) \in(0,1] \times(0,1]$, respectively, and $\tilde{a}=(a, a)$.

The rest of the paper is presented as follows: In Section 2, we include some necessary preliminaries needed in our proofs later. The main results are presented and proved in Section 3. Finally, two examples are provided to illustrate our main results.

\section{Fundamental Results}

In this section, we present some basic definitions and classical results about fractional calculus, matrix analysis, and fixed-point theorems that are be used throughout this paper.

Let $\tilde{I}:=[a, b] \times[a, c]$ and we consider $C(\tilde{I}, \mathbb{R})$ the Banach space of all continuous functions $x$ from $\tilde{I}$ into $\mathbb{R}$ with the following norm.

$$
\|x\|_{\infty}=\sup _{(\tau, \mathrm{u}) \in \tilde{\mathrm{I}}}|x(\tau, \mathrm{u})| .
$$

Next, we review some elementary properties of $\psi$-Riemann-Liouville fractional partial integral and the $\psi$-Caputo fractional partial derivative.

Definition 1 ([12]). Let $\tilde{a}=(a, a)$ and $\beta=\left(\beta_{1}, \beta_{2}\right)$, where $\beta_{1}, \beta_{2}>0$. Moreover, let $\psi(\cdot)$ be an increasing positive monotone function on each of $(a, b]$ and $(a, c]$ and allow it to have a continuous derivative $\psi^{\prime}(\cdot)$ on each of $(a, b]$ and $(a, c]$. The $\psi$-Riemann-Liouville partial integral of a function $x \in L^{1}(\tilde{I}, \mathbb{R})$ is defined as follows.

$$
\left(\mathbb{I}_{\tilde{a}}^{\beta ; \psi} x\right)(\tau, \mathrm{u})=\int_{a}^{\tau} \int_{a}^{\mathrm{u}} \frac{\psi^{\prime}(s) \psi^{\prime}(t)(\psi(\tau)-\psi(s))^{\beta_{1}-1}(\psi(\mathrm{u})-\psi(t))^{\beta_{2}-1}}{\Gamma\left(\beta_{1}\right) \Gamma\left(\beta_{2}\right)} x(s, t) \mathrm{dtds} .
$$

Definition 2 ([12]). Let $\tilde{a}=(a, a)$ and $\beta=\left(\beta_{1}, \beta_{2}\right)$, where $0<\beta_{1}, \beta_{2} \leq 1$. Moreover, let $x \in C^{1}(\tilde{\mathrm{I}}, \mathbb{R})$, and let $\psi$ belong to both $C^{1}([a, b], \mathbb{R})$ and $C^{1}([a, c], \mathbb{R})$ such that $\psi$ is increasing in both cases, and $\psi^{\prime}(\tau) \neq 0, \psi^{\prime}(\mathrm{u}) \neq 0$, for all $(\tau, \mathrm{u}) \in \tilde{\mathrm{I}}$. The $\psi$-Caputo fractional partial derivative of functions of two variables of order $\beta$ is given by the following.

$$
\left({ }^{c} \mathbb{D}_{\tilde{a}}^{\beta ; \psi} x\right)(\tau, \mathrm{u})=\left(\mathbb{I}_{\tilde{a}}^{1-\beta ; \psi} x\right)\left(\frac{1}{\psi^{\prime}(\tau) \psi^{\prime}(\mathrm{u})} \frac{\partial^{2}}{\partial \tau \partial \mathrm{u}}\right) x(\tau, \mathrm{u}) .
$$


Lemma 1 ([12]). Let $\sigma_{1}, \sigma_{2} \in(-1,+\infty)$ and $\beta=\left(\beta_{1}, \beta_{2}\right) \in(0, \infty) \times(0, \infty)$. Then, we have the following.

$$
\begin{aligned}
& \mathbb{I}_{\tilde{a}}^{\beta ; \psi}\left((\psi(\tau)-\psi(a))^{\sigma_{1}}(\psi(\mathbf{u})-\psi(a))^{\sigma_{2}}\right)=\frac{\Gamma\left(1+\sigma_{1}\right) \Gamma\left(1+\sigma_{2}\right)}{\Gamma\left(\beta_{1}+\sigma_{1}+1\right) \Gamma\left(\beta_{2}+\sigma_{2}+1\right)} \times \\
&(\psi(\tau)-\psi(a))^{\beta_{1}+\sigma_{1}}(\psi(\mathbf{u})-\psi(a))^{\beta_{2}+\sigma_{2}} .
\end{aligned}
$$

Lemma 2. Let $\tilde{a}=(a, a)$ and $\beta=\left(\beta_{1}, \beta_{2}\right)$ where $\beta=\left(\beta_{1}, \beta_{2}\right) \in(0, \infty) \times(0, \infty)$. Then, for all $(\tau, \mathrm{u}) \in \tilde{\mathrm{I}}$, we have the following.

$$
\mathbb{I}_{\tilde{a}}^{\beta ; \psi} e^{\theta(\psi(\tau)+\psi(\mathrm{u}))} \leq \frac{e^{\theta(\psi(\tau)+\psi(\mathrm{u}))}}{\theta^{\beta_{1}+\beta_{2}}} .
$$

Proof. From Definition 1, we have the following.

$\mathbb{I}_{\tilde{a}}^{\beta ; \psi} e^{\theta(\psi(\tau)+\psi(\mathrm{u}))}=\int_{a}^{\tau} \int_{a}^{\mathrm{u}} \frac{\psi^{\prime}(s) \psi^{\prime}(t)(\psi(\tau)-\psi(s))^{\beta_{1}-1}(\psi(\mathrm{u})-\psi(t))^{\beta_{2}-1}}{\Gamma\left(\beta_{1}\right) \Gamma\left(\beta_{2}\right)} e^{\theta(\psi(s)+\psi(t))} \mathrm{dtds}$.

Using the change of variables $\mathrm{y}=\psi(\tau)-\psi(s)$ and $\mathrm{z}=\psi(\mathrm{u})-\psi(t)$, we obtain the following.

$$
\mathbb{I}_{\tilde{a}}^{\beta ; \psi} e^{\lambda(\psi(\tau)+\psi(\mathrm{u}))}=\frac{e^{\theta(\psi(\tau)+\psi(\mathrm{u}))}}{\Gamma\left(\beta_{1}\right) \Gamma\left(\beta_{2}\right)} \int_{0}^{\psi(\tau)-\psi(a)} \int_{0}^{\psi(\mathrm{u})-\psi(a)} \mathrm{y}^{\beta_{1}-1} \mathrm{z}^{\beta_{2}-1} e^{-\theta(\mathrm{y}+\mathrm{z})} \mathrm{dzd} \mathrm{y} .
$$

Using now the change of variables $\mathrm{v}=\lambda \mathrm{y}$ and $\mathrm{w}=\lambda \mathrm{z}$ in the above equation, we obtain the following.

$$
\begin{aligned}
\mathbb{I}_{\tilde{a}}^{\beta ; \psi} e^{\theta(\psi(\tau)+\psi(\mathrm{u}))} & \leq \frac{e^{\theta(\psi(\tau)+\psi(\mathrm{u}))}}{\Gamma\left(\beta_{1}\right) \Gamma\left(\beta_{2}\right) \lambda^{\beta_{1}+\beta_{2}}} \int_{0}^{\infty} \int_{0}^{\infty} \mathrm{v}^{\beta_{1}-1} \mathrm{w}^{\beta_{2}-1} e^{-(\mathrm{v}+\mathrm{w})} \mathrm{dwdv} \\
& =\frac{e^{\theta(\psi(\tau)+\psi(\mathrm{u}))}}{\theta^{\beta_{1}+\beta_{2}}} .
\end{aligned}
$$

This completes the proof.

Let $x, y \in \mathbb{R}^{m}$ with $x=\left(x_{1}, x_{2}, \ldots, x_{m}\right), y=\left(y_{1}, y_{2}, \ldots, y_{m}\right)$.

By $x \leq y$, we mean $x_{i} \leq y_{i}, i=1, \ldots, m$. Moreover, the following is the case:

$$
\begin{gathered}
|x|=\left(\left|x_{1}\right|,\left|x_{2}\right|, \ldots,\left|x_{m}\right|\right), \\
\max (x, y)=\left(\max \left(x_{1}, y_{1}\right), \max \left(x_{2}, y_{2}\right), \ldots, \max \left(x_{m}, y_{m}\right)\right),
\end{gathered}
$$

and, thus, we obtain the following.

$$
\mathbb{R}_{+}^{m}=\left\{x \in \mathbb{R}^{m}: x_{i} \in \mathbb{R}_{+}, i=1, \ldots, m\right\} .
$$

If $c \in \mathbb{R}$, then $x \leq c$ means $x_{i} \leq c, i=1, \ldots, m$.

Definition 3 ([29]). Consider a nonempty set $\Xi$. A vector-valued metric on $\Xi$ refers to a map $\varkappa: \Xi \times \Xi \rightarrow \mathbb{R}^{m}$ that verifies the following:

(i) $\varkappa(\mathfrak{p}, \mathfrak{q}) \geq 0$ for all $\mathfrak{p}, \mathfrak{q} \in \Xi$, and if $\varkappa(\mathfrak{p}, \mathfrak{q})=0$, then $\mathfrak{p}=\mathfrak{q}$;

(ii) $\varkappa(\mathfrak{p}, \mathfrak{q})=\varkappa(\mathfrak{q}, \mathfrak{p})$ for all $\mathfrak{p}, \mathfrak{q} \in \Xi$;

(iii) $\varkappa(\mathfrak{p}, \mathfrak{w}) \leq \varkappa(\mathfrak{p}, \mathfrak{q})+\varkappa(\mathfrak{q}, \mathfrak{w})$ for all $\mathfrak{p}, \mathfrak{q}, \mathfrak{w} \in \Xi$. 
The pair $(\Xi, \varkappa)$ denotes a generalized metric space with the following.

$$
\varkappa(\mathfrak{p}, \mathfrak{q}):=\left(\begin{array}{c}
\varkappa_{1}(\mathfrak{p}, \mathfrak{q}) \\
\varkappa_{2}(\mathfrak{p}, \mathfrak{q}) \\
\vdots \\
\varkappa_{m}(\mathfrak{p}, \mathfrak{q})
\end{array}\right) .
$$

It should be noted that $\varkappa$ is a generalized metric space on $\Xi$ if and only if $\varkappa_{j}, j=1, \ldots, m$ are metrics on $\Xi$.

Definition 4 ([30]). A square matrix $\mathfrak{X}$ of real numbers is convergent to zero if and only if its spectral radius $\rho(\mathfrak{X})$ is strictly less than 1 .

Theorem 1 ([30]). For any nonnegative square matrix $\mathfrak{X}$, the following properties are equivalent:

1. $\mathfrak{X}$ is convergent to zero;

2. $\rho(\mathfrak{X})<1$;

3. Matrix $I-\mathfrak{X}$ is nonsingular and the following is the case;

$$
(I-\mathfrak{X})^{-1}=I+\mathfrak{X}+\cdots+\mathfrak{X}^{n}+\cdots ;
$$

4. $\quad I-\mathfrak{X}$ is nonsingular and $(I-\mathfrak{X})^{-1}$ is a nonnegative matrix where I denotes the unit matrix of $\mathfrak{X}_{m \times m}(\mathbb{R})$.

Example 1 ([31]). Matrix $\mathfrak{X} \in \mathfrak{X}_{2 \times 2}(\mathbb{R})$ given by the following:

$$
\mathfrak{X}=\left(\begin{array}{ll}
\kappa_{1} & \kappa_{2} \\
\kappa_{3} & \kappa_{4}
\end{array}\right)
$$

converges to zero in the following instances:

(1) $\kappa_{2}=\kappa_{3}=0, \kappa_{1}, \kappa_{4}>0$, and $\max \left\{\kappa_{1}, \kappa_{4}\right\}<1$;

(2) $\kappa_{3}=0, \kappa_{1}, \kappa_{4}>0, \kappa_{1}+\kappa_{4}<1$, and $-1<\kappa_{2}<0$;

(3) $\kappa_{1}+\kappa_{2}=\kappa_{3}+\kappa_{4}=0, \kappa_{1}>1, \kappa_{3}>0$, and $\left|\kappa_{1}-\kappa_{3}\right|<1$.

Definition $5([32,33])$. Let $(\Xi, \varkappa)$ be a generalized metric space. $\mathfrak{S}: \Xi \rightarrow \Xi$ is referred to as a contractive operator if there exists a matrix $\mathfrak{X}$ convergent to zero where the following is the case.

$$
\varkappa(\mathfrak{S}(\mathfrak{p}), \mathfrak{S}(\mathfrak{q})) \leq \mathfrak{X} \varkappa(\mathfrak{p}, \mathfrak{q}), \quad \text { for all } \mathfrak{p}, \mathfrak{q} \in \Xi .
$$

Now, we state the fixed-point theorems that are be employed in the sequel.

Theorem 2 ([32,34]). Let $(\Xi, \varkappa)$ be a complete generalized metric space and $\mathfrak{S}: \Xi \rightarrow \Xi$ be a contractive operator with Lipschitz matrix $\mathfrak{X}$. Then, $\mathfrak{S}$ has a unique fixed point $\mathfrak{p}_{0}$, and for each $\mathfrak{p} \in \Xi$, we have the following.

$$
\varkappa\left(\mathfrak{S}^{k}(\mathfrak{p}), \mathfrak{p}_{0}\right) \leq \mathfrak{X}^{k}(I-\mathfrak{X})^{-1} \varkappa(\mathfrak{p}, \mathfrak{S}(\mathfrak{p})) \quad \text { for all } k \in \mathbb{N} \text {. }
$$

Theorem 3 ([29]). Let $\Omega$ be a closed, convex, non-empty subset of generalized Banach spaces $\Xi$. Assume that $\mathbb{G}$ and $\mathbb{H}$ map $\Omega$ into $\Xi$ and that the following is the case:

(i) $\mathbb{G} \mathfrak{p}+\mathbb{H} \mathfrak{q} \in \Omega$ for all $\mathfrak{p}, \mathfrak{q} \in \Omega$;

(ii) $\mathbb{G}$ is compact and continuous;

(iii) $\mathbb{H}$ is an $\mathfrak{X}$-contraction mapping.

Then, $\mathbb{G} z+\mathbb{H} z=z$ has at least one solution on $\Omega$. 


\section{Existence, Uniqueness and Stability Results}

Definition 6. By a solution of problem (1)-(2), we mean a coupled function $(x, y) \in C(\tilde{I}, \mathbb{R}) \times$ $C(\tilde{I}, \mathbb{R})$ that satisfies the following system:

$$
\left\{\begin{array}{l}
\left({ }^{c} \mathbb{D}_{\tilde{a}}^{\alpha ; \psi} x\right)(\tau, \mathrm{u})=\lambda_{1} x(\tau, \mathrm{u})+\mathbb{F}_{1}(\tau, \mathrm{u}, x(\tau, \mathrm{u}), y(\tau, \mathrm{u})), \\
\left({ }^{c} \mathbb{D}_{\tilde{a}}^{\beta ; \psi} y\right)(\tau, \mathrm{u})=\lambda_{2} y(\tau, \mathrm{u})+\mathbb{F}_{2}(\tau, \mathrm{u}, x(\tau, \mathrm{u}), y(\tau, \mathrm{u})),
\end{array} \quad(\tau, \mathrm{u}) \in \tilde{\mathrm{I}},\right.
$$

and the initial conditions are the following.

$$
\left\{\begin{array}{l}
x(\tau, a)=\eta_{1}(\tau), \quad \tau \in[a, b], \\
x(a, \mathrm{u})=v_{1}(\mathrm{u}), \quad \mathrm{u} \in[a, c], \\
x(a, a)=\eta_{1}(a)=v_{1}(a), \\
y(\tau, a)=\eta_{2}(\tau), \quad \tau \in[a, b] \\
y(a, \mathrm{u})=v_{2}(\mathrm{u}), \quad \mathrm{u} \in[a, c], \\
y(a, a)=\eta_{2}(a)=v_{2}(a) .
\end{array}\right.
$$

Lemma 3. Let $f_{1}, f_{2} \in C(\tilde{I}, \mathbb{R})$. Then, the integral solution for the linear system of fractional differential equations is as follows:

$$
\left\{\begin{array}{l}
\left({ }^{c} \mathbb{D}_{\tilde{a}}^{\alpha ; \psi} x\right)(\tau, \mathrm{u})=f_{1}(\tau, \mathrm{u}), \\
\left({ }^{c} \mathbb{D}_{\tilde{a}}^{\beta ; \psi} y\right)(\tau, \mathrm{u})=f_{2}(\tau, \mathrm{u})
\end{array} \quad(\tau, \mathrm{u}) \in \tilde{\mathrm{I}}\right.
$$

which is supplemented with initial conditions (2). It is equivalent to the following integral equations:

$$
\left\{\begin{array}{l}
x(\tau, \mathrm{u})=\zeta_{1}(\tau, \mathrm{u})+\int_{a}^{\tau} \int_{a}^{\mathrm{u}} \frac{\psi^{\prime}(s) \psi^{\prime}(t)(\psi(\tau)-\psi(s))^{\alpha_{1}-1}(\psi(\mathrm{u})-\psi(t))^{\alpha_{2}-1}}{\Gamma\left(\alpha_{1}\right) \Gamma\left(\alpha_{2}\right)} f_{1}(s, t) \mathrm{dtds} \\
y(\tau, \mathrm{u})=\zeta_{2}(\tau, \mathrm{u})+\int_{a}^{\tau} \int_{a}^{\mathrm{u}} \frac{\psi^{\prime}(s) \psi^{\prime}(t)(\psi(\tau)-\psi(s))^{\beta_{1}-1}(\psi(\mathrm{u})-\psi(t))^{\beta_{2}-1}}{\Gamma\left(\beta_{1}\right) \Gamma\left(\beta_{2}\right)} f_{2}(s, t) \mathrm{dtds}
\end{array}\right.
$$

where the following is the case.

$$
\begin{aligned}
& \zeta_{1}(\tau, \mathrm{u})=\eta_{1}(\tau)+v_{1}(\mathrm{u})-\eta_{1}(a), \\
& \zeta_{2}(\tau, \mathrm{u})=\eta_{2}(\tau)+v_{2}(\mathrm{u})-\eta_{2}(a) .
\end{aligned}
$$

Proof. The proof is similar to the one provided in [16].

As a consequence of Lemma 3, we have the following result, which is useful for our main results.

Lemma 4. Let $\alpha=\left(\alpha_{1}, \alpha_{2}\right), \beta=\left(\beta_{1}, \beta_{2}\right) \in(0,1] \times(0,1]$ be fixed, $\lambda_{1}, \lambda_{2} \in \mathbb{R}_{+}$and $\mathbb{F}_{1}, \mathbb{F}_{2} \in C\left(\tilde{\mathrm{I}} \times \mathbb{R}^{2}, \mathbb{R}\right)$. Then, the coupled systems (1) and (2) is equivalent to the following integral equations.

$$
\left\{\begin{aligned}
x(\tau, \mathrm{u})= & \zeta_{1}(\tau, \mathrm{u})+\int_{a}^{\tau} \int_{a}^{\mathrm{u}} \frac{\psi^{\prime}(s) \psi^{\prime}(t)(\psi(\tau)-\psi(s))^{\alpha_{1}-1}(\psi(\mathrm{u})-\psi(t))^{\alpha_{2}-1}}{\Gamma\left(\alpha_{1}\right) \Gamma\left(\alpha_{2}\right)} \\
& \times\left(\lambda_{1} x(s, t)+\mathbb{F}_{1}(s, t, x(s, t), y(s, t))\right) \mathrm{dtds} \\
y(\tau, \mathrm{u})= & \zeta_{2}(\tau, \mathrm{u})+\int_{a}^{\tau} \int_{a}^{\mathrm{u}} \frac{\psi^{\prime}(s) \psi^{\prime}(t)(\psi(\tau)-\psi(s))^{\beta_{1}-1}(\psi(\mathrm{u})-\psi(t))^{\beta_{2}-1}}{\Gamma\left(\beta_{1}\right) \Gamma\left(\beta_{2}\right)} \\
& \times\left(\lambda_{2} y(s, t)+\mathbb{F}_{2}(s, t, x(s, t), y(s, t))\right) \mathrm{dtds} .
\end{aligned}\right.
$$

Our first result on the uniqueness is based on the Perov's fixed point theorem combined with the Bielecki norm.

Theorem 4. Let the following assumptions hold:

(H1) $\mathbb{F}_{1}, \mathbb{F}_{2}: \tilde{I} \times \mathbb{R}^{2} \rightarrow \mathbb{R}$ are continuous functions; 
(H2) There exist continuous functions $p_{i}, q_{i}: \tilde{\mathrm{I}} \rightarrow \mathbb{R}_{+}, i=1,2$, such that the following is the case:

$$
\left|\mathbb{F}_{i}\left(\tau, \mathrm{u}, x_{1}, y_{1}\right)-\mathbb{F}_{i}\left(\tau, \mathrm{u}, x_{2}, y_{2}\right)\right| \leq p_{i}(\tau, \mathrm{u})\left|x_{1}-x_{2}\right|+q_{i}(\tau, \mathrm{u})\left|y_{1}-y_{2}\right|
$$

for all $(\tau, \mathrm{u}) \in \tilde{\mathrm{I}}$ and each $\left(x_{1}, y_{1}\right),\left(x_{2}, y_{2}\right) \in \mathbb{R}^{2}$.

Then, the coupled system (1)-(2) has a unique solution.

For the sake of brevity, we set the following.

$$
\begin{gathered}
p_{i}^{*}:=\sup _{(\tau, \mathrm{u}) \in \tilde{\mathrm{I}}} p_{i}(\tau, \mathrm{u}), q_{i}^{*}:=\sup _{(\tau, \mathrm{u}) \in \tilde{\mathrm{I}}} q_{i}(\tau, \mathrm{u}), \zeta_{i}^{*}:=\sup _{(\tau, \mathrm{u}) \in \tilde{\mathrm{I}}}\left|\zeta_{i}(\tau, \mathrm{u})\right|, \mathbb{F}_{i}^{*}:=\sup _{(\tau, \mathrm{u}) \in \tilde{\mathrm{I}}}\left|\mathbb{F}_{i}(\tau, \mathrm{u}, 0,0)\right|, \\
\mathbb{M}_{\psi}^{1}:=\frac{(\psi(b)-\psi(a))^{\alpha_{1}}(\psi(\mathrm{c})-\psi(a))^{\alpha_{2}}}{\Gamma\left(\alpha_{1}+1\right) \Gamma\left(\alpha_{2}+1\right)}, \quad \mathbb{M}_{\psi}^{2}:=\frac{(\psi(b)-\psi(a))^{\beta_{1}}(\psi(\mathrm{c})-\psi(a))^{\beta_{2}}}{\Gamma\left(\beta_{1}+1\right) \Gamma\left(\beta_{2}+1\right)}
\end{gathered}
$$

Proof. Consider the Banach space $C(\tilde{\mathrm{I}}, \mathbb{R})$ equipped with a Bielecki norm type $\|\cdot\|_{\mathfrak{B}}$ defined below.

$$
\|x\|_{\mathfrak{B}}:=\sup _{(\tau, \mathbf{u}) \in \tilde{\mathrm{I}}} \frac{|x(\tau, \mathrm{u})|}{e^{\theta(\psi(\tau)+\psi(\mathrm{u}))}}, \quad \theta>0 .
$$

Consequently, the product space $\mathbb{X}:=C(\tilde{\mathrm{I}}, \mathbb{R}) \times C(\tilde{\mathrm{I}}, \mathbb{R})$ is a generalized Banach space endowed with the Bielecki vector-valued norm.

$$
\|(x, y)\|_{\mathbb{X}, \mathfrak{B}}=\left(\begin{array}{c}
\|x\|_{\mathfrak{B}} \\
\|y\|_{\mathfrak{B}}
\end{array}\right) .
$$

We define an operator $\mathfrak{S}=\left(\mathfrak{S}_{1}, \mathfrak{S}_{2}\right): \mathbb{X} \rightarrow \mathbb{X}$ by the following:

$$
\mathfrak{S}(x, y)=\left(\mathfrak{S}_{1}(x, y), \mathfrak{S}_{2}(x, y)\right) .
$$

where

$$
\begin{aligned}
\left(\mathfrak{S}_{1}(x, y)\right)(\tau, \mathrm{u})= & \zeta_{1}(\tau, \mathrm{u})+\int_{a}^{\tau} \int_{a}^{\mathrm{u}} \frac{\psi^{\prime}(s) \psi^{\prime}(t)(\psi(\tau)-\psi(s))^{\alpha_{1}-1}(\psi(\mathrm{u})-\psi(t))^{\alpha_{2}-1}}{\Gamma\left(\alpha_{1}\right) \Gamma\left(\alpha_{2}\right)} \\
& \times\left(\lambda_{1} x(s, t)+\mathbb{F}_{1}(s, t, x(s, t), y(s, t))\right) \mathrm{d} \mathrm{dds},
\end{aligned}
$$

and the following obtains.

$$
\begin{aligned}
\left(\mathfrak{S}_{2}(x, y)\right)(\tau, \mathrm{u})= & \zeta_{2}(\tau, \mathrm{u})+\int_{a}^{\tau} \int_{a}^{\mathrm{u}} \frac{\psi^{\prime}(s) \psi^{\prime}(t)(\psi(\tau)-\psi(s))^{\beta_{1}-1}(\psi(\mathrm{u})-\psi(t))^{\beta_{2}-1}}{\Gamma\left(\beta_{1}\right) \Gamma\left(\beta_{2}\right)} \\
& \times\left(\lambda_{2} y(s, t)+\mathbb{F}_{2}(s, t, x(s, t), y(s, t))\right) \mathrm{dtds} .
\end{aligned}
$$

Now, we apply Perov's fixed point theorem to prove that $\mathfrak{S}$ has a unique fixed point. Indeed, it sufficient to show that $\mathfrak{S}$ is a $\mathfrak{X}$-contraction mapping on $\mathbb{X}$ via the Bielecki's vector-valued norm. For this end, given $\left(x_{1}, y_{1}\right),\left(x_{2}, y_{2}\right) \in \mathbb{X}$ and $(\tau, \mathrm{u}) \in \tilde{\mathrm{I}}$, using $(\mathrm{H} 2)$ and Lemma 2, we can obtain the following. 


$$
\begin{aligned}
& \left|\left(\mathfrak{S}_{1}\left(x_{1}, y_{1}\right)\right)(\tau, \mathrm{u})-\left(\mathfrak{S}_{1}\left(x_{2}, y_{2}\right)\right)(\tau, \mathrm{u})\right| \\
\leq & \int_{a}^{\tau} \int_{a}^{\mathrm{u}} \frac{\psi^{\prime}(s) \psi^{\prime}(t)(\psi(\tau)-\psi(s))^{\alpha_{1}-1}(\psi(\mathrm{u})-\psi(t))^{\alpha_{2}-1}}{\Gamma\left(\alpha_{1}\right) \Gamma\left(\alpha_{2}\right)} \\
& \times\left(p_{1}(s, t)\left|x_{1}(s, t)-x_{2}(s, t)\right|+q_{1}(s, t)\left|y_{1}(s, t)-y_{2}(s, t)\right|\right) \mathrm{dtds} \\
+ & \lambda_{1} \int_{a}^{\tau} \int_{a}^{\mathrm{u}} \frac{\psi^{\prime}(s) \psi^{\prime}(t)(\psi(\tau)-\psi(s))^{\alpha_{1}-1}(\psi(\mathrm{u})-\psi(t))^{\alpha_{2}-1}}{\Gamma\left(\alpha_{1}\right) \Gamma\left(\alpha_{2}\right)}\left|x_{1}(s, t)-x_{2}(s, t)\right| \mathrm{d} \mathrm{dds} \\
\leq & \left(p_{1}^{*}\left\|x_{1}-x_{2}\right\|_{\mathfrak{B}}+q_{1}^{*}\left\|y_{1}-y_{2}\right\|_{\mathfrak{B}}+\lambda_{1}\left\|x_{1}-x_{2}\right\|_{\mathfrak{B}}\right) \\
& \times \int_{a}^{\tau} \int_{a}^{\mathrm{u}} \frac{\psi^{\prime}(s) \psi^{\prime}(t)(\psi(\tau)-\psi(s))^{\alpha_{1}-1}(\psi(\mathrm{u})-\psi(t))^{\alpha_{2}-1}}{\Gamma\left(\alpha_{1}\right) \Gamma\left(\alpha_{2}\right)} e^{\theta(\psi(s)+\psi(t))} \mathrm{dtds} \\
= & \frac{e^{\theta(\psi(\tau)+\psi(\mathrm{u}))}}{\theta^{\alpha_{1}+\alpha_{2}}}\left(p_{1}^{*}\left\|x_{1}-x_{2}\right\|_{\mathfrak{B}}+q_{1}^{*}\left\|y_{1}-y_{2}\right\|_{\mathfrak{B}}+\lambda_{1}\left\|x_{1}-x_{2}\right\|_{\mathfrak{B}}\right) .
\end{aligned}
$$

Hence, the following is concluded.

$$
\left\|\mathfrak{S}_{1}\left(x_{1}, y_{1}\right)-\mathfrak{S}_{1}\left(x_{2}, y_{2}\right)\right\|_{\mathfrak{B}} \leq \frac{p_{1}^{*}+\lambda_{1}}{\theta^{\alpha_{1}+\alpha_{2}}}\left\|x_{1}-x_{2}\right\|_{\mathfrak{B}}+\frac{q_{1}^{*}}{\theta^{\alpha_{1}+\alpha_{2}}}\left\|y_{1}-y_{2}\right\|_{\mathfrak{B}} .
$$

Furthermore, for each $\left(x_{1}, y_{1}\right),\left(x_{2}, y_{2}\right) \in \mathbb{X}$ and $(\tau, \mathrm{u}) \in \tilde{\mathrm{I}}$, we obtain the following.

$$
\left\|\mathfrak{S}_{2}\left(x_{1}, y_{1}\right)-\mathfrak{S}_{2}\left(x_{2}, y_{2}\right)\right\|_{\mathfrak{B}} \leq \frac{p_{2}^{*}}{\theta^{\beta_{1}+\beta_{2}}}\left\|x_{1}-x_{2}\right\|_{\mathfrak{B}}+\frac{q_{2}^{*}+\lambda_{2}}{\theta^{\beta_{1}+\beta_{2}}}\left\|y_{1}-y_{2}\right\|_{\mathfrak{B}} .
$$

This implies the following:

$$
\left\|\mathfrak{S}\left(x_{1}, y_{1}\right)-\mathfrak{S}\left(x_{2}, y_{2}\right)\right\|_{\mathbb{X}, \mathfrak{B}} \leq \mathfrak{X}\left\|\left(x_{1}, y_{1}\right)-\left(x_{2}, y_{2}\right)\right\|_{\mathbb{X}, \mathfrak{B}} .
$$

where the following is obtained.

$$
\mathfrak{X}=\left(\begin{array}{cc}
\frac{p_{1}^{*}+\lambda_{1}}{\theta^{\alpha}+\alpha_{2}} & \frac{q_{1}^{*}}{\theta_{1}+\alpha_{2}} \\
\frac{p_{2}^{*}}{\theta^{\beta}+\beta_{2}} & \frac{q_{2}^{*}+\lambda_{2}}{\theta^{\beta}+\beta_{2}}
\end{array}\right) .
$$

Taking $\theta$ that is large enough, it follows that matrix $\mathfrak{X}$ is convergent to zero and, thus, an application of Perov's theorem shows that $\mathfrak{S}$ has a unique fixed point. Thus, the coupled system (1)-(2) has a unique solution in $\mathbb{X}$.

Now, we provide our existence result for problem (1) and (2). The arguments are based on the Krasnoselskii-type fixed point theorem in generalized Banach spaces.

Theorem 5. Let the assumptions (H1) and (H2) be satisfied. Then, the coupled system (1) and (2) has at least one solution, provided that the spectral radius of matrix $\mathbb{K}_{\psi}$ is less than one, where matrix $\mathbb{K}_{\psi}$ is defined as follows.

$$
\mathbb{K}_{\psi}=\left(\begin{array}{cc}
\mathbb{M}_{\psi}^{1}\left(p_{1}^{*}+\lambda_{1}\right) & \mathbb{M}_{\psi}^{1} q_{1}^{*} \\
\mathbb{M}_{\psi}^{2} p_{2}^{*} & \mathbb{M}_{\psi}^{2}\left(q_{2}^{*}+\lambda_{2}\right)
\end{array}\right) .
$$

Proof. In order to use Krasnoselskii's fixed point theorem to prove our main result, we define a subset $\mathbb{B}_{\mu}$ of $\mathbb{X}$ by the following:

$$
\mathbb{B}_{\mu}=\left\{(x, y) \in \mathbb{X}:\|(x, y)\|_{\mathbb{X}, \infty} \leq \mu\right\},
$$


where $\mu:=\left(\mu_{1}, \mu_{2}\right) \in \mathbb{R}_{+}^{2}$ will be specified later and $\|\cdot\|_{\mathbb{X}, \infty}$ denotes the Chebyshev norm. Moreover, notice that $\mathbb{B}_{\mu}$ is a closed, convex and bounded subset of the generalized Banach space $\mathbb{X}$ and construct operators $\mathbb{G}=\left(\mathbb{G}_{1}, \mathbb{G}_{2}\right)$ and $\mathbb{H}=\left(\mathbb{H}_{1}, \mathbb{H}_{2}\right)$ on $\mathbb{B}_{\mu}$ as follows:

$$
\left\{\begin{aligned}
\mathbb{G}_{1}(x, y)(\tau, \mathrm{u})= & \int_{a}^{\tau} \int_{a}^{\mathrm{u}} \frac{\psi^{\prime}(s) \psi^{\prime}(t)(\psi(\tau)-\psi(s))^{\alpha_{1}-1}(\psi(\mathrm{u})-\psi(t))^{\alpha_{2}-1}}{\Gamma\left(\alpha_{1}\right) \Gamma\left(\alpha_{2}\right)} \\
& \times \mathbb{F}_{1}(s, t, x(s, t), y(s, t)) \mathrm{dtds}, \\
\mathbb{G}_{2}(x, y)(\tau, \mathrm{u})= & \int_{a}^{\tau} \int_{a}^{\mathrm{u}} \frac{\psi^{\prime}(s) \psi^{\prime}(t)(\psi(\tau)-\psi(s))^{\beta_{1}-1}(\psi(\mathrm{u})-\psi(t))^{\beta_{2}-1}}{\Gamma\left(\beta_{1}\right) \Gamma\left(\beta_{2}\right)} \\
& \times \mathbb{F}_{2}(s, t, x(s, t), y(s, t)) \mathrm{dtds},
\end{aligned}\right.
$$

where the following also applies.

$$
\left\{\begin{array}{l}
\mathbb{H}_{1}(x, y)(\tau, \mathrm{u})=\zeta_{1}(\tau, \mathrm{u})+\lambda_{1} \int_{a}^{\tau} \int_{a}^{\mathrm{u}} \frac{\psi^{\prime}(s) \psi^{\prime}(t)(\psi(\tau)-\psi(s))^{\alpha_{1}-1}(\psi(\mathrm{u})-\psi(t))^{\alpha_{2}-1}}{\Gamma\left(\alpha_{1}\right) \Gamma\left(\alpha_{2}\right)} x(s, t) \mathrm{dtd}, \\
\mathbb{H}_{2}(x, y)(\tau, \mathrm{u})=\zeta_{2}(\tau, \mathrm{u})+\lambda_{2} \int_{a}^{\tau} \int_{a}^{\mathrm{u}} \frac{\psi^{\prime}(s) \psi^{\prime}(t)(\psi(\tau)-\psi(s))^{\beta_{1}-1}(\psi(\mathrm{u})-\psi(t))^{\beta_{2}-1}}{\Gamma\left(\beta_{1}\right) \Gamma\left(\beta_{2}\right)} y(s, t) \mathrm{dtds} .
\end{array}\right.
$$

Obviously, both $\mathbb{G}$ and $\mathbb{H}$ are well defined due to (H1) and (H2). Furthermore, by Lemma 4, the operator forms of system (3) may be written as follows.

$$
(x, y)=\left(\mathbb{G}_{1}(x, y), \mathbb{G}_{2}(x, y)\right)+\left(\mathbb{H}_{1}(x, y), \mathbb{H}_{2}(x, y)\right):=\mathfrak{S}(x, y) .
$$

Thus, the fixed point of operator $\mathfrak{S}$ coincides with the solution of the coupled system (1)-(2). We shall prove that $\mathbb{G}$ and $\mathbb{H}$ satisfy all conditions of Theorem 3. For clarity, we will divide the remainder of the proof into several steps.

Step 1: $\mathbb{G}(x, y)+\mathbb{H}(\bar{x}, \bar{y}) \in \mathbb{B}_{\mu}$, for any $(x, y),(\bar{x}, \bar{y}) \in \mathbb{B}_{\mu}$. Indeed, for $(x, y),(\bar{x}, \bar{y}) \in \mathbb{X}$, and for each $(\tau, \mathrm{u}) \in \tilde{\mathrm{I}}$, from the definition of the operator $\mathbb{G}_{1}$ and assumption (H2), we can obtain the following.

$$
\begin{aligned}
& \left|\mathbb{G}_{1}(x, y)(\tau, \mathrm{u})\right| \leq \int_{a}^{\tau} \int_{a}^{\mathrm{u}} \frac{\psi^{\prime}(s) \psi^{\prime}(t)(\psi(\tau)-\psi(s))^{\alpha_{1}-1}(\psi(\mathrm{u})-\psi(t))^{\alpha_{2}-1}}{\Gamma\left(\alpha_{1}\right) \Gamma\left(\alpha_{2}\right)} \\
& \times\left(\left|\mathbb{F}_{1}(s, t, x(s, t), y(s, t))-\mathbb{F}_{1}(s, t, 0,0)\right|+\left|\mathbb{F}_{1}(s, t, 0,0)\right|\right) \mathrm{d} \mathrm{dds} \\
\leq & \left(p_{1}^{*}\|x\|_{\infty}+q_{1}^{*}\|y\|_{\infty}+\mathbb{F}_{1}^{*}\right) \int_{a}^{\tau} \int_{a}^{\mathrm{u}} \frac{\psi^{\prime}(s) \psi^{\prime}(t)(\psi(\tau)-\psi(s))^{\alpha_{1}-1}(\psi(\mathrm{u})-\psi(t))^{\alpha_{2}-1}}{\Gamma\left(\beta_{1}\right) \Gamma\left(\alpha_{2}\right)} \mathrm{dtds} \\
= & \mathbb{M}_{\psi}^{1}\left(p_{1}^{*}\|x\|_{\infty}+q_{1}^{*}\|y\|_{\infty}+\mathbb{F}_{1}^{*}\right) .
\end{aligned}
$$

Hence, the following is obtained.

$$
\left\|\mathbb{G}_{1}(x, y)\right\|_{\infty} \leq \mathbb{M}_{\psi}^{1}\left(p_{1}^{*}\|x\|_{\infty}+q_{1}^{*}\|y\|_{\infty}+\mathbb{F}_{1}^{*}\right) .
$$

By similar procedure, we obtain the following.

$$
\left\|\mathbb{G}_{2}(x, y)\right\|_{\infty} \leq \mathbb{M}_{\psi}^{2}\left(p_{2}^{*}\|x\|_{\infty}+q_{2}^{*}\|y\|_{\infty}+\mathbb{F}_{2}^{*}\right) .
$$

Thus, the above inequalities can be written in a vectorial form as follows:

$$
\|\mathbb{G}(x, y)\|_{\mathbb{X}, \infty}:=\left(\begin{array}{l}
\left\|\mathbb{G}_{1}(x, y)\right\|_{\infty} \\
\left\|\mathbb{G}_{2}(x, y)\right\|_{\infty}
\end{array}\right) \leq \mathbb{B}_{\psi}\left(\begin{array}{c}
\|x\|_{\infty} \\
\|y\|_{\infty}
\end{array}\right)+\left(\begin{array}{c}
\mathbb{M}_{\psi}^{1} \mathbb{F}_{1}^{*} \\
\mathbb{M}_{\psi}^{2} \mathbb{F}_{2}^{*}
\end{array}\right),
$$

where the following is obtained.

$$
\mathbb{B}_{\psi}=\left(\begin{array}{cc}
\mathbb{M}_{\psi}^{1} p_{1}^{*} & \mathbb{M}_{\psi}^{1} q_{1}^{*} \\
\mathbb{M}_{\psi}^{2} p_{2}^{*} & \mathbb{M}_{\psi}^{2} q_{2}^{*}
\end{array}\right)
$$


In a similar manner, we obtain the following:

$$
\|\mathbb{H}(\bar{x}, \bar{y})\|_{\mathbb{X}, \infty}:=\left(\begin{array}{c}
\left\|\mathbb{H}_{1}(\bar{x}, \bar{y})\right\|_{\infty} \\
\left\|\mathbb{H}_{2}(\bar{x}, \bar{y})\right\|_{\infty}
\end{array}\right) \leq \mathbb{D}_{\psi}\left(\begin{array}{c}
\|\bar{x}\|_{\infty} \\
\|\bar{y}\|_{\infty}
\end{array}\right)+\left(\begin{array}{c}
\zeta_{1}^{*} \\
\zeta_{2}^{*}
\end{array}\right)
$$

where the following is concluded.

$$
\mathbb{D}_{\psi}=\left(\begin{array}{cc}
\lambda_{1} \mathbb{M}_{\psi}^{1} & 0 \\
0 & \lambda_{2} \mathbb{M}_{\psi}^{2}
\end{array}\right)
$$

By combining (11) and (12), we obtain the following.

$$
\|\mathbb{H}(\bar{x}, \bar{y})\|_{\mathbb{X}, \infty}+\|\mathbb{G}(x, y)\|_{\mathbb{X}, \infty} \leq \mathbb{B}_{\psi}\left(\begin{array}{c}
\|x\|_{\infty} \\
\|y\|_{\infty}
\end{array}\right)+\mathbb{D}_{\psi}\left(\begin{array}{c}
\|\bar{x}\|_{\infty} \\
\|\bar{y}\|_{\infty}
\end{array}\right)+\left(\begin{array}{c}
\mathbb{M}_{\psi}^{1} \mathbb{F}_{1}^{*}+\zeta_{1}^{*} \\
\mathbb{M}_{\psi}^{2} \mathbb{F}_{2}^{*}+\zeta_{2}^{*}
\end{array}\right)
$$

Now, we search for $\mu=\left(\mu_{1}, \mu_{2}\right) \in \mathbb{R}_{+}^{2}$ such that $\mathbb{G}(x, y)+\mathbb{H}(\bar{x}, \bar{y}) \in \mathbb{B}_{\mu}$ for any $(x, y),(\bar{x}, \bar{y}) \in \mathbb{B}_{\mu}$. To this end, according to (13), it is sufficient to show the following:

$$
\mathbb{K}_{\psi}\left(\begin{array}{c}
\mu_{1} \\
\mu_{2}
\end{array}\right)+\left(\begin{array}{c}
\mathbb{M}_{\psi}^{1} \mathbb{F}_{1}^{*}+\zeta_{1}^{*} \\
\mathbb{M}_{\psi}^{2} \mathbb{F}_{2}^{*}+\zeta_{2}^{*}
\end{array}\right) \leq\left(\begin{array}{l}
\mu_{1} \\
\mu_{2}
\end{array}\right)
$$

where the following is the case.

$$
\mathbb{K}_{\psi}=\mathbb{B}_{\psi}+\mathbb{D}_{\psi}
$$

Equivalently, we can also obtain the following.

$$
\left(\begin{array}{c}
\mathbb{M}_{\psi}^{1} \mathbb{F}_{1}^{*}+\zeta_{1}^{*} \\
\mathbb{M}_{\psi}^{2} \mathbb{F}_{2}^{*}+\zeta_{2}^{*}
\end{array}\right) \leq\left(I-\mathbb{K}_{\psi}\right)\left(\begin{array}{c}
\mu_{1} \\
\mu_{2}
\end{array}\right)
$$

Since matrix $\mathbb{K}_{\psi}$ is convergent to zero, it is known, from Theorem 3, that matrix $\left(I-\mathbb{K}_{\psi}\right)$ is nonsingular and $\left(I-\mathbb{K}_{\psi}\right)^{-1}$ has non-negative elements. Therefore, (14) is equivalent to the following:

$$
\left(\begin{array}{l}
\mu_{1} \\
\mu_{2}
\end{array}\right) \geq\left(I-\mathbb{K}_{\psi}\right)^{-1}\left(\begin{array}{l}
\mathbb{M}_{\psi}^{1} \mathbb{F}_{1}^{*}+\zeta_{1}^{*} \\
\mathbb{M}_{\psi}^{2} \mathbb{F}_{2}^{*}+\zeta_{2}^{*}
\end{array}\right) .
$$

which means that $\mathbb{G}(x, y)+\mathbb{H}(\bar{x}, \bar{y}) \in \mathbb{B}_{\mu}$.

Step 2: $\mathbb{H}$ is a $\tilde{\mathbb{D}}_{\psi}$-contraction mapping on $\mathbb{B}_{\mu}$. In fact, for each $(\tau, \mathrm{u}) \in \tilde{\mathrm{I}}$ and for any $\left(x_{1}, y_{1}\right),\left(x_{2}, y_{2}\right) \in \mathbb{B}_{\mu}$. By the same method of the proof of Theorem 4 , we can easily show the following:

$$
\left\|\mathbb{H}\left(x_{1}, y_{1}\right)-\mathbb{H}\left(x_{2}, y_{2}\right)\right\|_{\mathbb{X}, \mathfrak{B}} \leq \tilde{\mathbb{D}}_{\psi}\left\|\left(x_{1}, y_{1}\right)-\left(x_{2}, y_{2}\right)\right\|_{\mathbb{X}, \mathfrak{B}}
$$

where the following is the case.

$$
\tilde{\mathbb{D}}_{\psi}=\left(\begin{array}{cc}
\frac{\lambda_{1}}{\theta^{\alpha_{1}+\alpha_{2}}} & 0 \\
0 & \frac{\lambda_{2}}{\theta^{\beta_{1}+\beta_{2}}}
\end{array}\right) .
$$

Taking $\theta$ to be large enough, it follows that matrix $\tilde{\mathbb{D}}_{\psi}$ is convergent to zero; thus, $\mathbb{H}$ is an $\tilde{\mathbb{D}}_{\psi}$-contraction mapping on $\mathbb{B}_{\mu}$ with respect to the Bielecki norm. 
Step 3: $\mathbb{G}$ is compact and continuous. Firstly, the continuity of $\mathbb{G}$ follows from the continuity of $\mathbb{F}_{1}$ and $\mathbb{F}_{2}$. Next, we prove that $\mathbb{G}$ is uniformly bounded on $\mathbb{B}_{\mu}$. From (11) and for each $(x, y) \in \mathbb{B}_{\mu}$, we can obtain the following.

$$
\|\mathbb{G}(x, y)\|_{\mathbb{X}, \infty}:=\left(\begin{array}{l}
\left\|\mathbb{G}_{1}(x, y)\right\|_{\infty} \\
\left\|\mathbb{G}_{2}(x, y)\right\|_{\infty}
\end{array}\right) \leq \mathbb{B}_{\psi}\left(\begin{array}{c}
\mu_{1} \\
\mu_{2}
\end{array}\right)+\left(\begin{array}{c}
\mathbb{M}_{\psi}^{1} \mathbb{F}_{1}^{*} \\
\mathbb{M}_{\psi}^{2} \mathbb{F}_{2}^{*}
\end{array}\right)<\infty
$$

This proves that $\mathbb{G}$ is uniformly bounded.

Finally, we show that $\mathbb{G}\left(\mathbb{B}_{\mu}\right)$ is equicontinuous. Let $(x, y) \in \mathbb{B}_{\mu}$ and any $\left(\tau_{1}, \mathrm{u}_{1}\right)$, $\left(\tau_{2}, \mathrm{u}_{2}\right) \in \tilde{\mathrm{I}}$, with $\tau_{1}<\tau_{2}$ and $\mathrm{u}_{1}<\mathrm{u}_{2}$. Taking (H2) into consideration we can find the following.

$$
\begin{aligned}
& \left|\mathbb{G}_{1}(x, y)\left(\tau_{2}, \mathrm{u}_{2}\right)-\mathbb{G}_{1}(x, y)\left(\tau_{1}, \mathrm{u}_{1}\right)\right| \\
& \leq \frac{p_{1}^{*} \mu_{1}+q_{1}^{*} \mu_{2}+\mathbb{F}_{1}^{*}}{\left.\Gamma \alpha_{1}\right) \Gamma\left(\alpha_{2}\right)} \int_{a}^{\tau_{1}} \int_{a}^{u_{1}} \psi^{\prime}(s) \psi^{\prime}(t)\left[\left(\psi\left(\tau_{1}\right)-\psi(s)\right)^{\alpha_{1}-1}\left(\psi\left(\mathrm{u}_{1}\right)-\psi(t)\right)^{\alpha_{2}-1}\right. \\
& \left.-\left(\psi\left(\tau_{2}\right)-\psi(s)\right)^{\alpha_{1}-1}\left(\psi\left(\mathbf{u}_{2}\right)-\psi(t)\right)^{\alpha_{2}-1}\right] \mathrm{dtds} \\
& +\frac{p_{1}^{*} \mu_{1}+q_{1}^{*} \mu_{2}+\mathbb{F}_{1}^{*}}{\Gamma\left(\alpha_{1}\right) \Gamma\left(\alpha_{2}\right)} \int_{\tau_{1}}^{\tau_{2}} \int_{\mathrm{u}_{1}}^{\mathrm{u}_{2}} \psi^{\prime}(s) \psi^{\prime}(t)\left(\psi\left(\tau_{2}\right)-\psi(s)\right)^{\alpha_{1}-1}\left(\psi\left(\mathrm{u}_{2}\right)-\psi(t)\right)^{\alpha_{2}-1} \mathrm{dtd} s \\
& +\frac{p_{1}^{*} \mu_{1}+q_{1}^{*} \mu_{2}+\mathbb{F}_{1}^{*}}{\Gamma\left(\alpha_{1}\right) \Gamma\left(\alpha_{2}\right)} \int_{\tau_{1}}^{\tau_{2}} \int_{a}^{\mathrm{u}_{1}} \psi^{\prime}(s) \psi^{\prime}(t)\left(\psi\left(\tau_{2}\right)-\psi(s)\right)^{\alpha_{1}-1}\left(\psi\left(\mathbf{u}_{2}\right)-\psi(t)\right)^{\alpha_{2}-1} \mathrm{dtd} s \\
& +\frac{p_{1}^{*} \mu_{1}+q_{1}^{*} \mu_{2}+\mathbb{F}_{1}^{*}}{\Gamma\left(\alpha_{1}\right) \Gamma\left(\alpha_{2}\right)} \int_{a}^{\tau_{1}} \int_{\mathrm{u}_{1}}^{\mathrm{u}_{2}} \psi^{\prime}(s) \psi^{\prime}(t)\left(\psi\left(\tau_{2}\right)-\psi(s)\right)^{\alpha_{1}-1}\left(\psi\left(\mathrm{u}_{2}\right)-\psi(t)\right)^{\alpha_{2}-1} \mathrm{dtd} s \\
& \leq \frac{p_{1}^{*} \mu_{1}+q_{1}^{*} \mu_{2}+\mathbb{F}_{1}^{*}}{\Gamma\left(\alpha_{1}+1\right) \Gamma\left(\alpha_{2}+1\right)}\left[2\left(\psi\left(\mathrm{u}_{2}\right)-\psi\left(\mathrm{u}_{1}\right)\right)^{\alpha_{2}}\left[\left(\psi\left(\tau_{2}\right)-\psi(a)\right)^{\alpha_{1}}-\left(\psi\left(\tau_{2}\right)-\psi\left(\tau_{1}\right)\right)^{\alpha_{1}}\right]\right. \\
& \left.+\left[\left(\psi\left(\mathbf{u}_{2}\right)-\psi(a)\right)^{\alpha_{2}}\left(\psi\left(\tau_{2}\right)-\psi\left(\tau_{1}\right)\right)^{\alpha_{1}}-\left(\psi\left(\mathbf{u}_{1}\right)-\psi(a)\right)^{\alpha_{2}}\left(\psi\left(\tau_{1}\right)-\psi(a)\right)^{\alpha_{1}}\right]\right] .
\end{aligned}
$$

Similarly, the following can be obtained.

$$
\begin{aligned}
& \left|\mathbb{G}_{2}(x, y)\left(\tau_{2}, \mathrm{u}_{2}\right)-\mathbb{G}_{2} x\left(\tau_{1}, \mathrm{u}_{1}\right)\right| \\
\leq & \frac{p_{1}^{*} \mu_{1}+q_{1}^{*} \mu_{2}+\mathbb{F}_{1}^{*}}{\Gamma\left(\beta_{1}+1\right) \Gamma\left(\beta_{2}+1\right)}\left[2\left(\psi\left(\mathrm{u}_{2}\right)-\psi\left(\mathrm{u}_{1}\right)\right)^{\beta_{2}}\left[\left(\psi\left(\tau_{2}\right)-\psi(a)\right)^{\beta_{1}}-\left(\psi\left(\tau_{2}\right)-\psi\left(\tau_{1}\right)\right)^{\beta_{1}}\right]\right. \\
& \left.+\left[\left(\psi\left(\mathrm{u}_{2}\right)-\psi(a)\right)^{\beta_{2}}\left(\psi\left(\tau_{2}\right)-\psi\left(\tau_{1}\right)\right)^{\beta_{1}}-\left(\psi\left(\mathrm{u}_{1}\right)-\psi(a)\right)^{\beta_{2}}\left(\psi\left(\tau_{1}\right)-\psi(a)\right)^{\beta_{1}}\right]\right] .
\end{aligned}
$$

As $\tau_{1} \rightarrow \tau_{2}$ and $\mathrm{u}_{1} \rightarrow \mathrm{u}_{2}$, the right-hand side of the above inequalities tends to zero independently of $(x, y) \in \mathbb{B}_{\mu}$. Hence, the operators $\mathbb{G}_{1}$ and $\mathbb{G}_{2}$ are equicontinuous; thus, the operator $\mathbb{G}$ is equicontinuous. By Arzelà-Ascoli's theorem, we deduce that $\mathbb{G}$ is a compact operator. Thus, all the assumptions of Theorem 3 are satisfied. As a consequence of Krasnoselskii's fixed point theorem, we conclude that operator $\mathfrak{S}=\mathbb{G}+\mathbb{H}$ defined by (10) has at least one fixed point $(x, y) \in \mathbb{B}_{\mu}$, which is just the solution of system (1) and (2). This completes the proof of the Theorem 5.

Now, We complete this section by studying Ulam-Hyers stability for problem (1)-(2) by means of integral representation of its solution given by the following:

$$
x(\tau, \mathrm{u})=\mathfrak{S}_{1}(x, y)(\tau, \mathrm{u}), \quad y(t)=\mathfrak{S}_{2}(x, y)(\tau, \mathrm{u}),
$$

where $\mathfrak{S}_{1}$ and $\mathfrak{S}_{2}$ are defined by (6) and (7).

Define the following nonlinear operators $\mathbb{S}_{1}, \mathbb{S}_{2}: \mathbb{X} \rightarrow C(\tilde{\mathrm{I}}, \mathbb{R})$.

$$
\left\{\begin{array}{l}
\left({ }^{c} \mathbb{D}_{\tilde{a}}^{\alpha ; \psi} \tilde{x}\right)(\tau, \mathrm{u})-\lambda_{1} \tilde{x}(\tau, \mathrm{u})-\mathbb{F}_{1}(\tau, \mathrm{u}, \tilde{x}(\tau, \mathrm{u}), \tilde{y}(\tau, \mathrm{u}))=\mathbb{S}_{1}(\tilde{x}, \tilde{y})(\tau, \mathrm{u}), \\
\left({ }^{c} \mathbb{D}_{\tilde{a}}^{\beta ; \psi} \tilde{y}\right)(\tau, \mathrm{u})-\lambda_{2} \tilde{y}(\tau, \mathrm{u})-\mathbb{F}_{2}(\tau, \mathrm{u}, \tilde{x}(\tau, \mathrm{u}), \tilde{y}(\tau, \mathrm{u}))=\mathbb{S}_{2}(\tilde{x}, \tilde{y})(\tau, \mathrm{u}),
\end{array} \quad(\tau, \mathrm{u}) \in \tilde{\mathrm{I}},\right.
$$


For some $\varepsilon_{1}, \varepsilon_{2}>0$, we consider the following inequality.

$$
\left\{\begin{array}{l}
\left|\mathbb{S}_{1}(\tilde{x}, \tilde{y})(\tau, \mathrm{u})\right| \leq \varepsilon_{1} \\
\left|\mathbb{S}_{2}(\tilde{x}, \tilde{y})(\tau, \mathrm{u})\right| \leq \varepsilon_{2}
\end{array} \quad(\tau, \mathrm{u}) \in \tilde{\mathrm{I}}\right.
$$

Definition 7 ([27,35]). The coupled system (1) and (2) is Ulam-Hyers stable if we can find a positive constants $a, b, c$, and $d$ such that for every $\varepsilon_{1}, \varepsilon_{1}>0$ and for each solution $(\tilde{x}, \tilde{y}) \in \mathbb{X}$ of inequality (15), there exists a solution $(x, y) \in \mathbb{X}$ of (1) and (2) with the following.

$$
\left\{\begin{array}{l}
|\tilde{x}(\tau, \mathrm{u})-x(\tau, \mathrm{u})| \leq a \varepsilon_{1}+b \varepsilon_{2}, \\
|\tilde{y}(\tau, \mathrm{u})-y(\tau, \mathrm{u})| \leq c \varepsilon_{1}+d \varepsilon_{2},
\end{array} \quad(\tau, \mathrm{u}) \in \tilde{\mathrm{I}} .\right.
$$

Theorem 6. Let the assumptions of Theorem 4 hold. Then, problem (1) and (2) is Ulam-Hyers stable with respect to Bielecki's norm.

Proof. Let $(x, y) \in \mathbb{X}$ be the solution of problem (1) and (2) satisfying (6) and (7). Let $(\tilde{x}, \tilde{y})$ be any solution satisfying (15).

$$
\left\{\begin{array}{l}
\left({ }^{c} \mathbb{D}_{\tilde{a}}^{\alpha ; \psi} \tilde{x}\right)(\tau, \mathrm{u})=\lambda_{1} \tilde{x}(\tau, \mathrm{u})+\mathbb{F}_{1}(\tau, \mathrm{u}, \tilde{x}(\tau, \mathrm{u}), \tilde{y}(\tau, \mathrm{u}))+\mathbb{S}_{1}(\tilde{x}, \tilde{y})(\tau, \mathrm{u}), \\
\left({ }^{c} \mathbb{D}_{\tilde{a}}^{\beta ;} \tilde{y}\right)(\tau, \mathrm{u})=\lambda_{2} \tilde{y}(\tau, \mathrm{u})+\mathbb{F}_{2}(\tau, \mathrm{u}, \tilde{x}(\tau, \mathrm{u}), \tilde{y}(\tau, \mathrm{u}))+\mathbb{S}_{2}(\tilde{x}, \tilde{y})(\tau, \mathrm{u}) .
\end{array} \quad(\tau, \mathrm{u}) \in \tilde{\mathrm{I}},\right.
$$

Thus, the following is obtained:

$$
\begin{aligned}
\tilde{x}(\tau, \mathrm{u})= & \mathfrak{S}_{1}(\tilde{x}, \tilde{y})(\tau, \mathrm{u})+\int_{a}^{\tau} \int_{a}^{\mathrm{u}} \frac{\psi^{\prime}(s) \psi^{\prime}(t)(\psi(\tau)-\psi(s))^{\alpha_{1}-1}(\psi(\mathrm{u})-\psi(t))^{\alpha_{2}-1}}{\Gamma\left(\alpha_{1}\right) \Gamma\left(\alpha_{2}\right)} \\
& \times \mathbb{S}_{1}(\tilde{x}, \tilde{y})(s, t) \mathrm{dtds},
\end{aligned}
$$

and the following is also the case.

$$
\begin{aligned}
\tilde{y}(\tau, \mathrm{u})= & \mathfrak{S}_{2}(\tilde{x}, \tilde{y})(\tau, \mathrm{u})+\int_{a}^{\mathrm{u}} \frac{\psi^{\prime}(s) \psi^{\prime}(t)(\psi(\tau)-\psi(s))^{\beta_{1}-1}(\psi(\mathrm{u})-\psi(t))^{\beta_{2}-1}}{\Gamma\left(\beta_{1}\right) \Gamma\left(\beta_{2}\right)} \\
& \times \mathbb{S}_{2}(\tilde{x}, \tilde{y})(s, t) \mathrm{dtds},
\end{aligned}
$$

It follows from (16) and (17) that we obtain the following:

$$
\begin{aligned}
\left|\tilde{x}(\tau, \mathrm{u})-\mathfrak{S}_{1}(\tilde{x}, \tilde{y})(\tau, \mathrm{u})\right| \leq & \int_{a}^{\tau} \int_{a}^{\mathrm{u}} \frac{\psi^{\prime}(s) \psi^{\prime}(t)(\psi(\tau)-\psi(s))^{\alpha_{1}-1}(\psi(\mathrm{u})-\psi(t))^{\alpha_{2}-1}}{\Gamma\left(\alpha_{1}\right) \Gamma\left(\alpha_{2}\right)} \\
& \times\left|\mathbb{S}_{1}(\tilde{x}, \tilde{y})(s, t)\right| \mathrm{dtds} \\
\leq & \mathbb{M}_{\psi}^{1} \varepsilon_{1}
\end{aligned}
$$

and the following inequality results.

$$
\begin{aligned}
\left|\tilde{y}(\tau, \mathrm{u})-\mathfrak{S}_{2}(\tilde{x}, \tilde{y})(\tau, \mathrm{u})\right| \leq & \int_{a}^{\tau} \int_{a}^{\mathrm{u}} \frac{\psi^{\prime}(s) \psi^{\prime}(t)(\psi(\tau)-\psi(s))^{\beta_{1}-1}(\psi(\mathrm{u})-\psi(t))^{\beta_{2}-1}}{\Gamma\left(\beta_{1}\right) \Gamma\left(\beta_{2}\right)} \\
& \times\left|\mathbb{S}_{2}(\tilde{x}, \tilde{y})(s, t)\right| \mathrm{dtds} \\
\leq & \mathbb{M}_{\psi}^{2} \varepsilon_{2}
\end{aligned}
$$

Thus, by (H2), Lemma 2 and inequalities (18), (19), we obtain the following.

$$
\begin{aligned}
|\tilde{x}(\tau, \mathrm{u})-x(\tau, \mathrm{u})| & =\left|\tilde{x}(\tau, \mathrm{u})-\mathfrak{S}_{1}(\tilde{x}, \tilde{y})(\tau, \mathrm{u})+\mathfrak{S}_{1}(\tilde{x}, \tilde{y})(\tau, \mathrm{u})-x(\tau, \mathrm{u})\right| \\
& \leq\left|\tilde{x}(\tau, \mathrm{u})-\mathfrak{S}_{1}(\tilde{x}, \tilde{y})(\tau, \mathrm{u})\right|+\left|\mathfrak{S}_{1}(\tilde{x}, \tilde{y})(\tau, \mathrm{u})-\mathfrak{S}_{1} x(\tau, \mathrm{u})\right| \\
& \leq \mathbb{M}_{\psi}^{1} \varepsilon_{1}+\left(\frac{p_{1}^{*}+\lambda_{1}}{\theta^{\alpha_{1}+\alpha_{2}}}\|\tilde{x}-x\|_{\mathfrak{B}}+\frac{q_{1}^{*}}{\theta^{\alpha_{1}+\alpha_{2}}}\|\tilde{y}-y\|_{\mathfrak{B}}\right) e^{\theta(\psi(\tau)+\psi(\mathrm{u}))} .
\end{aligned}
$$


Hence, we derive the following.

$$
\|\tilde{x}-x\|_{\mathfrak{B}} \leq \mathbb{M}_{\psi}^{1} \varepsilon_{1}+\frac{p_{1}^{*}+\lambda_{1}}{\theta^{\alpha_{1}+\alpha_{2}}}\|\tilde{x}-x\|_{\mathfrak{B}}+\frac{q_{1}^{*}}{\theta^{\alpha_{1}+\alpha_{2}}}\|\tilde{y}-y\|_{\mathfrak{B}} .
$$

Similarly, we have the following.

$$
\|\tilde{y}-y\|_{\mathfrak{B}} \leq \mathbb{M}_{\psi}^{2} \varepsilon_{2}+\frac{p_{2}^{*}}{\theta^{\beta_{1}+\beta_{2}}}\|\tilde{x}-x\|_{\mathfrak{B}}+\frac{q_{1}^{*}+\lambda_{2}}{\theta^{\beta_{1}+\beta_{2}}}\|\tilde{y}-y\|_{\mathfrak{B}} .
$$

Inequalities (20) and (21) can be rewritten in matrix form as follows:

$$
(I-\mathfrak{X})\left(\begin{array}{c}
\|\tilde{x}-x\|_{\mathfrak{B}} \\
\|\tilde{y}-y\|_{\mathfrak{B}}
\end{array}\right) \leq\left(\begin{array}{c}
\mathbb{M}_{\psi}^{1} \varepsilon_{1} \\
\mathbb{M}_{\psi}^{2} \varepsilon_{2}
\end{array}\right) .
$$

where $\mathfrak{X}$ is the matrix provided by (8). Since matrix $\mathfrak{X}$ is convergent to zero for sufficiently large $\theta$, it follows, from Theorem 3 , that matrix $(I-\mathfrak{X})$ is nonsingular and $(I-\mathfrak{X})^{-1}$ has nonnegative elements. Thus, (22) is equivalent to the following.

$$
\left(\begin{array}{c}
\|\tilde{x}-x\|_{\mathfrak{B}} \\
\|\tilde{y}-y\|_{\mathfrak{B}}
\end{array}\right) \leq(I-\mathfrak{X})^{-1}\left(\begin{array}{c}
\mathbb{M}_{\psi}^{1} \varepsilon_{1} \\
\mathbb{M}_{\psi}^{2} \varepsilon_{2}
\end{array}\right)
$$

Furthermore, if we denote the following:

$$
(I-\mathfrak{X})^{-1}=\left(\begin{array}{cc}
\kappa_{1} & \kappa_{2} \\
\kappa_{3} & \kappa_{4}
\end{array}\right) .
$$

then, (23) becomes the following:

$$
\left\{\begin{array}{l}
\|\tilde{x}-x\|_{\mathfrak{B}} \leq \kappa_{1} \mathbb{M}_{\psi}^{1} \varepsilon_{1}+\kappa_{2} \mathbb{M}_{\psi}^{2} \varepsilon_{2} \\
\|\tilde{y}-y\|_{\mathfrak{B}} \leq \kappa_{3} \mathbb{M}_{\psi}^{1} \varepsilon_{1}+\kappa_{4} \mathbb{M}_{\psi}^{2} \varepsilon_{2}
\end{array}\right.
$$

which means that the coupled system (1) and (2) is Ulam-Hyers stable with respect to Bielecki's norm $\|\cdot\|_{\mathbb{X}, \mathfrak{B}}$.

\section{Applications}

In this section, we provide two examples to illustrate our above results.

Example 2. Consider the following coupled system:

$$
\left\{\begin{array}{l}
\left({ }^{c} \mathbb{D}_{\tilde{a}}^{\alpha} x\right)(\tau, \mathrm{u})=x(\tau, \mathrm{u})+\mathbb{F}_{1}(\tau, \mathrm{u}, x(\tau, \mathrm{u}), y(\tau, \mathrm{u})), \\
\left({ }^{c} \mathbb{D}_{\tilde{a}}^{\beta} y\right)(\tau, \mathrm{u})=y(\tau, \mathrm{u})+\mathbb{F}_{2}(\tau, \mathrm{u}, x(\tau, \mathrm{u}), y(\tau, \mathrm{u})),
\end{array} \quad(\tau, \mathrm{u}) \in \tilde{\mathrm{I}}:=[0,1] \times[0,1],\right.
$$

with initial conditions of the following:

$$
\left\{\begin{array}{l}
x(\tau, 0)=\tau, \quad \tau \in[0,1], \\
x(0, \mathrm{u})=\mathrm{u}^{2}, \quad \mathrm{u} \in[0,1], \\
x(0,0)=0, \\
y(\tau, 0)=\tau e^{\tau}, \quad \tau \in[0,1], \\
y(0, \mathrm{u})=\mathrm{u}, \quad \mathrm{u} \in[0,1], \\
y(0,0)=0,
\end{array}\right.
$$

where

$$
\alpha=\left(\alpha_{1}, \alpha_{2}\right)=\beta=\left(\beta_{1}, \beta_{2}\right)=(0.75,0.75), \lambda_{1}=\lambda_{2}=1, a=0, b=c=1, \psi(t)=t .
$$


and the following is the case.

$$
\begin{aligned}
& \mathbb{F}_{1}(\tau, \mathrm{u}, x(\tau, \mathrm{u}), y(\tau, \mathrm{u}))=e^{\tau+\mathrm{u}} \ln (1+|x(\tau, \mathrm{u})|)+e^{\tau \mathrm{u}} \arctan y(\tau, \mathrm{u}), \\
& \mathbb{F}_{2}(\tau, \mathrm{u}, x(\tau, \mathrm{u}), y(\tau, \mathrm{u}))=\frac{\tau+\mathrm{u}}{(1+|x(\tau, \mathrm{u})|+|y(\tau, \mathrm{u})|)} .
\end{aligned}
$$

Clearly, the functions $\mathbb{F}_{1}$ and $\mathbb{F}_{2}$ are continuous. Moreover, for any $x, y \in \mathbb{R}$ and $(\tau, \mathrm{u}) \in \tilde{\mathrm{I}}$, we have the following:

$$
\begin{aligned}
& \left|\mathbb{F}_{1}\left(\tau, \mathrm{u}, x_{1}, y_{1}\right)-\mathbb{F}_{1}\left(\tau, \mathrm{u}, x_{2}, y_{2}\right)\right| \leq p_{1}(\tau, \mathrm{u})\left|x_{1}-x_{2}\right|+q_{1}(\tau, \mathrm{u})\left|y_{1}-y_{2}\right| \\
& \left|\mathbb{F}_{2}\left(\tau, \mathrm{u}, x_{1}, y_{1}\right)-\mathbb{F}_{2}\left(\tau, \mathrm{u}, x_{2}, y_{2}\right)\right| \leq p_{2}(\tau, \mathrm{u})\left|x_{1}-x_{2}\right|+q_{2}(\tau, \mathrm{u})\left|y_{1}-y_{2}\right|,
\end{aligned}
$$

where the following is the case.

$$
\begin{gathered}
p_{1}(\tau, \mathrm{u})=e^{\tau+\mathrm{u}}, \quad q_{1}(\tau, \mathrm{u})=e^{\tau \mathrm{u}}, \quad p_{2}(\tau, \mathrm{u})=q_{2}(\tau, \mathrm{u})=\tau+\mathrm{u} . \\
p_{1}^{*}:=e^{2}, q_{1}^{*}:=e, p_{2}^{*}=q_{2}^{*}:=2,
\end{gathered}
$$

Furthermore, matrix $\mathfrak{X}$ given by (8) has the following form.

$$
\mathfrak{X}=\frac{1}{\theta \sqrt{\theta}}\left(\begin{array}{cc}
e^{2}+1 & e \\
2 & 3
\end{array}\right) .
$$

Taking $\theta$ as large enough, it follows that matrix $\mathfrak{X}$ is convergent to zero; thus, an application of Theorem 4 shows that the coupled system (24) and (25) has a unique solution and is UlamHyers stable.

Example 3. Consider the following coupled system:

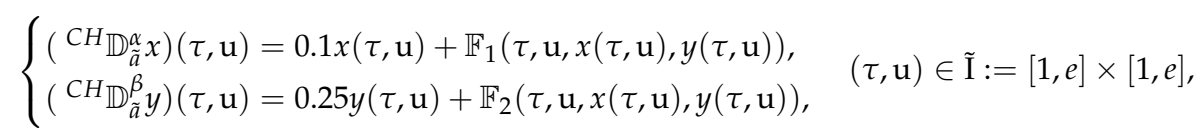

with initial conditions:

$$
\begin{cases}x(\tau, 1)=\tau, & \tau \in[1, e], \\ x(1, \mathrm{u})=\mathrm{u}, \quad \mathrm{u} \in[1, e], \\ x(1,1)=1, & \\ y(\tau, 1)=\tau, & \tau \in[1, e], \\ y(1, \mathrm{u})=\mathrm{u}, \quad \mathrm{u} \in[1, e], \\ y(1,1)=1, & \end{cases}
$$

where

$$
\begin{aligned}
\alpha & =\left(\alpha_{1}, \alpha_{2}\right)=\beta=\left(\beta_{1}, \beta_{2}\right)=(0.5,0.5), \psi(\cdot)=\ln (\cdot), a=1, b=c=e, \\
\lambda_{1} & =0.1, \lambda_{2}=0.25, \zeta_{1}^{*}=, \zeta_{2}^{*}=2 e-1, \quad \mathbb{M}_{\psi}^{1}=\mathbb{M}_{\psi}^{2}=\frac{4}{\pi} .
\end{aligned}
$$

and the following is the case.

$$
\begin{aligned}
& \mathbb{F}_{1}(\tau, \mathrm{u}, x(\tau, \mathrm{u}), y(\tau, \mathrm{u}))=\frac{x(\tau, \mathrm{u})}{e^{\tau+\mathrm{u}}(1+|x(\tau, \mathrm{u})|)}+\frac{y(\tau, \mathrm{u})}{\tau+\mathrm{u}+2^{\prime}} \\
& \mathbb{F}_{2}(\tau, \mathrm{u}, x(\tau, \mathrm{u}), y(\tau, \mathrm{u}))=\frac{1}{(\tau+\mathrm{u})^{2}}\left(\frac{\left.x(\tau, \mathrm{u})+\sqrt{1+x^{2}(\tau, \mathrm{u})}\right)}{2}+\sin |y(\tau, \mathrm{u})|\right) .
\end{aligned}
$$


Clearly, the functions $\mathbb{F}_{1}, \mathbb{F}_{2}$ are continuous. Moreover, for any $x, y \in \mathbb{R}$ and $(\tau, \mathrm{u}) \in \tilde{\mathrm{I}}$, we have the following:

$$
\begin{aligned}
& \left|\mathbb{F}_{1}\left(\tau, \mathrm{u}, x_{1}, y_{1}\right)-\mathbb{F}_{1}\left(\tau, \mathrm{u}, x_{2}, y_{2}\right)\right| \leq p_{1}(\tau, \mathrm{u})\left|x_{2}-x_{2}\right|+q_{1}(\tau, \mathrm{u})\left|y_{1}-y_{2}\right| \\
& \left|\mathbb{F}_{2}\left(\tau, \mathrm{u}, x_{1}, y_{1}\right)-\mathbb{F}_{2}\left(\tau, \mathrm{u}, x_{2}, y_{2}\right)\right| \leq p_{2}(\tau, \mathrm{u})\left|x_{1}-x_{2}\right|+q_{2}(\tau, \mathrm{u})\left|y_{1}-y_{2}\right|,
\end{aligned}
$$

where the following obtains.

$$
\begin{gathered}
p_{1}(\tau, \mathrm{u})=\frac{1}{e^{\tau+\mathrm{u}}}, \quad q_{1}(\tau, \mathrm{u})=\frac{1}{\tau+\mathrm{u}+2}, \quad p_{2}(\tau, \mathrm{u})=q_{2}(\tau, \mathrm{u})=\frac{1}{(\tau+\mathrm{u})^{2}} . \\
p_{1}^{*}=e^{-2}, \quad q_{1}^{*}=0.25, \quad p_{2}^{*}=0.25, \quad q_{2}^{*}=0.25 .
\end{gathered}
$$

Furthermore, matrix $\mathbb{K}_{\psi}$ given by (9) has the following form.

$$
\mathbb{K}_{\psi}=\frac{4}{\pi}\left(\begin{array}{cc}
e^{-2}+0.1 & 0.25 \\
0.25 & 0.5
\end{array}\right) .
$$

By using the MATLAB program, we can obtain the eigenvalues of $\mathbb{K}_{\psi}$ as follows: $\sigma_{1}=0.1080$, $\sigma_{2}=0.8283$. This shows that $\mathbb{K}_{\psi}$ is converging to zero. Therefore, by Theorem 5 , the coupled system (26) and (27) has at least one solution.

Author Contributions: Conceptualization, Z.B. and M.B.; formal analysis, Z.B. and C.D.; investigation, Z.B. and C.D.; writing-review and editing, M.B. and Y.Z. All authors have read and agreed to the published version of the manuscript.

Funding: This research was funded by Fundo para o Desenvolvimento das Ciências e da Tecnologia of Macau grant number 0074/2019/A2.

Institutional Review Board Statement: Not applicable.

Informed Consent Statement: Not applicable.

Data Availability Statement: No data was reported in this study.

Conflicts of Interest: The authors declare no conflict of interest.

\section{References}

1. Abbas, S.; Benchohra, M.; N'Guérxexkata, G.M. Developments in Mathematics. In Topics in Fractional Differential Equations; Springer: New York, NY, USA, 2012; Volume 27.

2. Abbas, S.; Benchohra, M.; N'Guerekata, G.M. Mathematics Research Developments. In Advanced Fractional Differential and Integral Equations; Nova Science Publishers, Inc.: New York, NY, USA, 2015.

3. Abbas, S.; Benchohra, M.; Graef, J.R.; Henderson, J. De Gruyter Series in Nonlinear Analysis and Applications. In Implicit Fractional Differential and Integral Equations; De Gruyter: Berlin, Germany, 2018; Volume 26.

4. Hilfer, R. Applications of Fractional Calculus in Physics; World Scientific Publishing Co., Inc.: River Edge, NJ, USA, 2000.

5. Kilbas, A.A.; Srivastava, H.M.; Trujillo, J.J. North-Holland Mathematics Studies. In Theory and Applications of Fractional Differential Equations; Elsevier Science B.V.: Amsterdam, The Netherlands, 2006; Volume 204.

6. Miller, K.S.; Ross, B. An Introduction to the Fractional Calculus and Fractional Differential Equations; A Wiley-Interscience Publication; John Wiley \& Sons, Inc.: New York, NY, USA, 1993.

7. Podlubny, I. Fractional Differential Equations; Mathematics in Science and Engineering; Academic Press, Inc.: San Diego, CA, USA, 1999; Volume 198.

8. Sabatier, J.; Agrawal, O.P.; Machado, J.A.T. Advances in Fractional Calculus; Springer: Dordrecht, The Netherlands, 2007.

9. Tarasov, V.E. Fractional Dynamics; Nonlinear Physical Science; Springer: Heidelberg, Germany, 2010.

10. Almeida, R. A Caputo fractional derivative of a function with respect to another function. Commun. Nonlinear Sci. Numer. Simul. 2017, 44, 460-481. [CrossRef]

11. Sousa, J.V.D.; de Oliveira, E.C. On the $\psi$-Hilfer fractional derivative. Commun. Nonlinear Sci. Numer. Simul. 2018, 60, 72-91. [CrossRef]

12. Sousa, J.V.D.C.; de Oliveira, E.C. On the Stability of a Hyperbolic Fractional Partial Differential Equation. Differ. Equ. Dyn. Syst. 2019. [CrossRef]

13. Abbas, S.; Benchohra, M.; Hamidi, N.; Henderson, J. Caputo-Hadamard fractional differential equations in Banach spaces. Fract. Calc. Appl. Anal. 2018, 21, 1027-1045. [CrossRef] 
14. Shikhare, P.U.; Kucche, K.D. Existence, uniqueness and Ulam stabilities for nonlinear hyperbolic partial integrodifferential equations. Int. J. Appl. Comput. Math. 2019, 5, 1-21. [CrossRef]

15. Vityuk, A.N.; Golushkov, A.V. Existence of solutions of systems of partial differential equations of fractional order. Nonlinear Oscil. 2004, 7, 318-325. [CrossRef]

16. Vivek, D.; Elsayed, E.M.; Kanagarajan, K. Theory and analysis of partial differential equations with a $\psi-$ Caputo fractional derivative. Rocky Mt. J. Math. 2019, 49, 1355-1370. [CrossRef]

17. Wang, J.R.; Feckan, M.; Zhou, Y. Fractional order differential switched systems with coupled nonlocal initial and impulsive conditions. Bull. Des Sci. Math. 2017, 141, 727-746. [CrossRef]

18. Zhou, Y.; He, J.W. New results on controllability of fractional evolution systems with order $\alpha \in(1,2)$. Evol. Equ. Control. Theory 2021, 10, 491-509. [CrossRef]

19. Zhou, Y.; He, J.W.; Ahmad, B.; Tuan, N.H. Existence and regularity results of a backward problem for fractional diffusion equations. Math. Meth. Appl. Sci. 2019, 42, 6775-6790. [CrossRef]

20. Chen, Y.; An, H.-L. Numerical solutions of coupled Burgers equations with time- and space-fractional derivatives. Appl. Math Comput. 2008, 200, 87-95. [CrossRef]

21. Fu, L.; Chen, Y.; Yang, H. Time-space fractional coupled generalized zakharov-kuznetsov equations set for rossby solitary waves in two-layer fluids. Mathematics 2019, 7, 41. [CrossRef]

22. Gafiychuk, V.; Datsko, B.; Meleshko, V. Mathematical modeling of time fractional reaction-diffusion systems. J. Comput. Appl. Math. 2008, 220, 215-225. [CrossRef]

23. Ahmad, M.; Zada, A.; Alzabut, J. Hyers-Ulam stability of a coupled system of fractional differential equations of Hilfer-Hadamard type. Demonstr. Math. 2019, 52, 283-295. [CrossRef]

24. Harikrishnan, S.; Shah, K.; Kanagarajan, K. Existence theory of fractional coupled differential equations via $\Psi$-Hilfer fractional derivative. Random Oper. Stoch. Equ. 2019, 27, 207-212. [CrossRef]

25. Liang, J.; Liu, Z.; Wang, X. Solvability for a couple system of nonlinear fractional differential equations in a Banach space. Fract. Calc. Appl. Anal. 2013, 16, 51-63. [CrossRef]

26. Mahmudov, N.I.; Al-Khateeb, A. Existence and Ulam-Hyers stability of coupled sequential fractional differential equations with integral boundary conditions. J. Inequal. Appl. 2019, 2019, 165. [CrossRef]

27. Zada, A.; Yar, M.; Li, T. Existence and stability analysis of nonlinear sequential coupled system of Caputo fractional differential equations with integral boundary conditions. Ann. Univ. Paedagog. Crac. Stud. Math. 2018, 17, 103-125. [CrossRef]

28. Zhang, Y.; Wang, J. Nonlocal Cauchy problems for a class of implicit impulsive fractional relaxation differential systems. J. Appl. Math. Comput. 2016, 52, 323-343. [CrossRef]

29. Petre, I.-R.; Petruşel, A. Krasnoselskii's theorem in generalized Banach spaces and applications. Electron. J. Qual. Theory Differ. Equ. 2012, 85, 1-20. [CrossRef]

30. Varga, R.S. Matrix Iterative Analysis, 2nd ed.; Springer Series in Computational Mathematics; Springer: Berlin, Germany, 2000; Volume 27.

31. Precup, R. The role of matrices that are convergent to zero in the study of semilinear operator systems. Math. Comput. Model. 2009, 49, 703-708. [CrossRef]

32. Precup, R.; Viorel, A. Existence results for systems of nonlinear evolution equations. Int. J. Pure Appl. Math. 2008, 47, 199-206.

33. Rus, I.A. Generalized Contractions and Applications; Cluj University Press: Cluj, Romania, 2001.

34. Perov, A.I. On the Cauchy problem for a system of ordinary differential equations. Pviblizhen. Met. Reshen. Differ. Uvavn. Vyp. 1964, 2, 115-134.

35. Urs, C. Coupled fixed point theorems and applications to periodic boundary value problems. Miskolc Math. Notes 2013, 14, 323-333. [CrossRef] 